\title{
WEIGHTED NORM INEQUALITIES FOR THE FOURIER TRANSFORM ON CERTAIN TOTALLY DISCONNECTED GROUPS
}

\author{
T. S. QUEK
}

\begin{abstract}
Let $G$ be a locally compact totally disconnected Abelian group with dual group $\Gamma$. Let $U$ and $V$ be nonnegative measurable functions on $\Gamma$ and $G$, respectively. In this paper we give, in terms of $U$ and $V$, a necessary condition and some sufficient conditions for the inequality $\|\hat{f} U\|_{q} \leq C\|f V\|_{p}$ to hold for all $f$ in $L_{1}(G)$, where $\hat{f}$ denotes the Fourier transform of $f$ and $1<p \leq q<\infty$. If $U$ and $V$ are both radial, we give a necessary and sufficient condition for the above norm inequality to hold for all $f$ in $L_{1}(G)$.
\end{abstract}

1. Introduction. In $1978 \mathrm{~B}$. Muckenhoupt [6] posed the problem of characterizing, for given $p$ and $q$ with $1<p, q<\infty$, those nonnegative weight functions $U$ and $V$ on the real line $\mathbf{R}$ so that the inequality

$$
\|\hat{f} U\|_{q} \leq C\|f V\|_{p}
$$

holds for all $f$ in $L_{1}(\mathbf{R})$. This problem has been studied by, among others, Muckenhoupt himself $[\mathbf{7}, \mathbf{8}]$, W. B. Jurkat and G. Sampson [5], and H. P. Heinig and his coauthors J. J. Benedetto and R. Johnson $[1,2,3]$. The most elegant result for this problem is due to Benedetto, Heinig, and Johnson [2] who proved that for nonnegative radial weight functions $U$ and $V$ such that $U$ and $V^{-1}$ are even and decreasing on $(0, \infty)$, inequality $(1.1)$ is equivalent to

$$
\sup _{s>0}\left(\int_{0}^{s} U(x)^{q} d x\right)^{1 / q}\left(\int_{0}^{1 / s} V(x)^{-p^{\prime}} d x\right)^{1 / p^{\prime}}<\infty
$$

where $1 / p+1 / p^{\prime}=1$.

Recently C. W. Onneweer [10] has studied Muckenhoupt's problem for functions defined on certain locally compact Abelian groups $G$. He considers mainly the case in which the weight functions $U$ and $V$ are such that $U$ and $V^{-1}$ are in certain weak $L_{p}$-spaces. In this paper we also consider Muckenhoupt's problem for functions defined on $G$. Theorem 1.1 gives a necessary condition for inequality (1.1) to hold for all $f$ in $L_{1}(G)$; it is an analogue on $G$ of Theorem 2 of Benedetto and Heinig [1]. In Theorems 1.2 and 1.3 we obtain a sufficient condition for inequality (1.1) to hold for all $f$ in $L_{1}(G)$. Our results closely resemble Theorems 1, 3, and 4 of Muckenhoupt [7] for functions in $L_{1}(\mathbf{R})$. The main result of this paper is Theorem 1.4 in which we give a necessary and sufficient condition (see (1.5)) for inequality (1.1) to hold for all $f$ in $L_{1}(G)$, under the assumption that the weight functions $U$ and $V$ are radial (the definition of radial function will be given later in this section). We remark that Theorem 1.4 resembles the Benedetto-Heinig-Johnson result mentioned

Received by the editors June 11, 1986.

1980 Mathematics Subject Classification (1985 Revision). Primary 43A25; Secondary 43A15. 
earlier. Indeed, condition (1.5)(i) is an obvious replacement on $G$ of condition (1.2), whereas condition (1.5)(ii) replaces their monotonicity assumptions on the weight functions.

The remainder of this section is devoted to a brief discussion of the class of groups considered here and statements of our results. Proofs of these results will be given in subsequent sections.

Throughout this paper $G$ will denote a locally compact Abelian group with a two-way infinite sequence $\left(G_{n}\right)_{-\infty}^{\infty}$ of compact open subgroups such that

(i) $G_{n+1} \varsubsetneqq G_{n}$ for every $n \in \mathbf{Z}=\{\ldots,-2,-1,0,1,2, \ldots\}$;

(ii) $\bigcup_{n=-\infty}^{\infty} G_{n}=G$ and $\bigcap_{n=-\infty}^{\infty} G_{n}=\{0\}$;

(iii) $\sup \left\{\operatorname{order}\left(G_{n} / G_{n+1}\right): n \in \mathbf{Z}\right\}<\infty$.

The dual group of $G$ will be denoted by $\Gamma$, and the annihilator of $G_{n}$ will be denoted by $\Gamma_{n}$. Thus $\left(\Gamma_{n}\right)_{-\infty}^{\infty}$ is a sequence of compact open subgroups of $\Gamma$ such that

(i') $\Gamma_{n} \varsubsetneqq \Gamma_{n+1}$ for every $n \in \mathbf{Z}$;

(ii') $\bigcup_{n=-\infty}^{\infty} \Gamma_{n}=\Gamma$ and $\bigcap_{n=-\infty}^{\infty} \Gamma_{n}=\{1\}$.

The Haar measure $\mu$ on $G$ and the Haar measure $\lambda$ on $\Gamma$ are chosen so that $\mu\left(G_{0}\right)=1=\lambda\left(\Gamma_{0}\right)$. Then $\left(\mu\left(G_{n}\right)\right)^{-1}=\lambda\left(\Gamma_{n}\right)$ and we denote this number by $m_{n}$.

We define a metric $d$ on $G \times G$ by

$$
d(x, y)= \begin{cases}0 & \text { if } x=y, \\ \left(m_{n}\right)^{-1} & \text { if } x-y \in G_{n} \backslash G_{n+1} .\end{cases}
$$

The topology on $G$ induced by this metric is the same as the original topology on $G$. For $x \in G$ we write $\|x\|$ for $d(x, 0)$; then $\|x\|=\left(m_{n}\right)^{-1}$ if and only if $x \in G_{n} \backslash G_{n+1}$. In a similar way we can define a metric $\tilde{d}$ on $\Gamma \times \Gamma$; if we set $\|\gamma\|=\tilde{d}(\gamma, 1)$, then $\|\gamma\|=m_{n}$ if and only if $\gamma \in \Gamma_{n+1} \backslash \Gamma_{n}$. A function $f$ on $G$ is called radial if $f(x)=f(\|x\|)$; thus, a radial function on $G$ is constant on each subset $G_{n} \backslash G_{n+1}$ in $G(n \in \mathbf{Z})$. A similar definition can be given for radial functions on $\Gamma$.

For $p$ with $1<p<\infty, p^{\prime}$ will denote the number such that $1 / p+1 / p^{\prime}=1$. The characteristic function of the set $E$ is denoted by $\xi_{E}$. The symbols ${ }^{\wedge}$ and $\vee$ will be used to denote the Fourier transform and the inverse Fourier transform, respectively. The conventions $0 \cdot \infty=0, \sup \varnothing=-\infty$, and $\inf \varnothing=\infty$ will also be used. As usual, $C$ will denote a generic constant, which may assume different values in different places.

We now state the results obtained in this paper.

THEOREM 1.1. Let $1<p, q<\infty$ and let $U, V$ be nonnegative measurable functions on $\Gamma$ and $G$, respectively. Then a necessary condition for inequality (1.1) to hold for all $f$ in $L_{1}(G)$ is that

$$
\sup _{n \in \mathbf{Z}}\left(\int_{\Gamma_{n}} U(\gamma)^{q} d \lambda\right)^{1 / q}\left(\int_{G_{n}} V(x)^{-p^{\prime}} d \mu\right)^{1 / p^{\prime}}<\infty .
$$

THEOREM 1.2. Let $1<p \leq q \leq p^{\prime}$ and let $U, V$ be nonnegative measurable functions on $\Gamma$ and $G$, respectively. Then a sufficient condition for inequality (1.1) to hold for all $f$ in $L_{1}(G)$ is that

$$
\sup _{r>0}\left(\int_{U^{\#}(\gamma)>B r} U(\gamma)^{q} d \lambda\right)^{1 / q}\left(\int_{V(x)<r} V(x)^{-p^{\prime}} d \mu\right)^{1 / p^{\prime}}<\infty
$$


where $B$ is a positive constant and $U^{\#}$ is defined on $\Gamma$ by $U^{\#}(\gamma)=U(\gamma)\|\gamma\|^{1 / \beta}, \beta$ being defined by $1 / \beta=1 / p+1 / q-1$.

THEOREM 1.3. Let $1<q^{\prime} \leq p \leq q$ and let $U, V$ be nonnegative measurable functions on $\Gamma$ and $G$, respectively. Then a sufficient condition for inequality (1.1) to hold for all $f$ in $L_{1}(G)$ is that

$$
\sup _{r>0}\left(\int_{V^{\#}(x)>B r} V(x)^{-p^{\prime}} d \mu\right)^{1 / p^{\prime}}\left(\int_{U(\gamma)^{-1}<r} U(\gamma)^{q} d \lambda\right)^{1 / q}<\infty
$$

for some positive constant $B$, where $V^{\#}$ is defined on $G$ by

$$
V^{\#}(x)=\left(V(x)\|x\|^{1 / \beta}\right)^{-1}
$$

$\beta$ being defined by $1 / \beta=1 / p+1 / q-1$.

THEOREM 1.4. Let $1<p \leq q<\infty$ and let $U, V$ be nonnegative radial functions on $\Gamma$ and $G$, respectively. Then a necessary and sufficient condition for inequality (1.1) to hold for all $f$ in $L_{1}(G)$ is that

$$
\left\{\begin{array}{l}
\text { (i) } \sup _{n \in \mathbf{Z}}\left(\int_{\Gamma_{n}} U(\gamma)^{q} d \lambda\right)^{1 / q}\left(\int_{G_{n}} V(x)^{-p^{\prime}} d \mu\right)^{1 / p^{\prime}}<\infty ; \\
\text { (ii) } \sup _{n \in \mathbf{Z}}\left(\sup _{i<n} V_{i}^{-1} U_{n}\left(m_{n}\right)^{1 / q-1 / p^{\prime}}\right)<\infty,
\end{array}\right.
$$

where $U_{n}=U(\gamma)$ when $\gamma \in \Gamma_{n} \backslash \Gamma_{n-1}$ and $V_{n}=V(x)$ when $x \in G_{n} \backslash G_{n+1}$.

2. Proof of Theorem 1.1. Let $1<p, q<\infty$ and let $N$ be any fixed integer. For $k \in \mathbf{Z}^{+}=\{1,2,3, \ldots\}$, define the function $f_{N, k}$ on $G$ by

$$
f_{N, k}(x)= \begin{cases}V(x)^{-p^{\prime}} & \text { if } x \in G_{N} \text { and } V(x)^{-p^{\prime}} \leq k \\ k & \text { if } x \in G_{N} \text { and } V(x)^{-p^{\prime}}>k \\ 0 & \text { otherwise. }\end{cases}
$$

Note that $\gamma(x)=1$ if $\gamma \in \Gamma_{N}$ and $x \in G_{N}$. Hence for $\gamma \in \Gamma_{N}$, we have

$$
\hat{f}_{N, k}(\gamma)=\int_{G_{N}} f_{N, k}(x) d \mu=\int_{A_{N, k}} V(x)^{-p^{\prime}} d \mu+\int_{B_{N, k}} k d \mu
$$

where $A_{N, k}=\left\{x \in G_{N}: V(x)^{-p^{\prime}} \leq k\right\}$ and $B_{N, k}=\left\{x \in G_{N}: V(x)^{-p^{\prime}}>k\right\}$. Now

$$
\begin{aligned}
& \left(\int_{\Gamma_{N}} U(\gamma)^{q}\left|\int_{G_{N}} f_{N, k}(x) d \mu\right|^{q} d \lambda\right)^{1 / q} \\
& \quad=\left(\int_{\Gamma_{N}} U(\gamma)^{q}\left|\hat{f}_{N, k}(\gamma)\right|^{q} d \lambda\right)^{1 / q} \leq\left\|\hat{f}_{N, k} U\right\|_{q} \leq C\left\|f_{N, k} V\right\|_{p} \\
& =C\left(\int_{A_{N, k}} V(x)^{-p^{\prime} p} V(x)^{p} d \mu+\int_{B_{N, k}} k^{p} V(x)^{p} d \mu\right)^{1 / p} \\
& \leq C\left(\int_{A_{N, k}} V(x)^{-p^{\prime}} d \mu+\int_{B_{N, k}} k d \mu\right)^{1 / p}\left(\text { since } V(x)^{p} \leq k^{-p / p^{\prime}} \text { for } x \in B_{N, k}\right) \\
& \quad=C\left(\int_{G_{N}} f_{N, k}(x) d \mu\right)^{1 / p} .
\end{aligned}
$$


Thus we get

$$
\left(\int_{\Gamma_{N}} U(\gamma)^{q} d \lambda\right)^{1 / q}\left(\int_{G_{N}} f_{N, k}(x) d \mu\right)^{1 / p^{\prime}} \leq C .
$$

Since $f_{N, k}(x) \rightarrow V(x)^{-p^{\prime}}$ on $G_{N}$, we have

$$
\left(\int_{\Gamma_{N}} U(\gamma)^{q} d \lambda\right)^{1 / q}\left(\int_{G_{N}} V(x)^{-p^{\prime}} d \mu\right)^{1 / p^{\prime}} \leq C .
$$

Noting that $C$ is independent of $N$, we complete the proof of Theorem 1.1.

3. Definitions and notation. In this section we establish some more definitions and notation which will facilitate our discussion in the proof of Theorem 1.2 in $\S 4$.

Throughout this section $f$ is a simple function on $G$ with support of finite measure such that $\|f\|_{1}=1$. Let $H=\{x \in G: f(x) \neq 0\}$. Let $V$ be any nonnegative measurable function on $G$ and define $V^{*}=\left\|V \xi_{H}\right\|_{\infty}$. If $V^{*}=\infty$, then one of the following is true:

(i) there exists a positive integer $K$ such that

$$
\int_{\{x \in H: V(x)>K\}}|f(x)| d \mu \leq 1 / 2,
$$

(ii) there exists a positive $\varepsilon$ such that $\mu\{x \in H: V(x)>k\} \geq \varepsilon$ for all $k \in \mathbf{Z}^{+}$. Thus $\mu\{x \in H: V(x)=\infty\} \geq \varepsilon$. Hence we have $\|\hat{f} U\|_{q} \leq\|f V\|_{p}=\infty$ for all $U$, where $1<p, q<\infty$.

Therefore we may assume that (i) happens whenever $V^{*}=\infty$. We now define $V_{1}^{*}$ by

$$
V_{1}^{*}= \begin{cases}V^{*} & \text { if } V^{*}<\infty \\ K & \text { if } V^{*}=\infty\end{cases}
$$

where $K$ is defined as in (i). For $i \in \mathbf{Z}^{+}$we define $V_{i+1}^{*}$ from $V_{i}^{*}$ by

$$
V_{i+1}^{*}=\left\|V \xi_{H \backslash\left(A_{0} \cup A_{1} \cup \cdots \cup A_{i}\right)}\right\|_{\infty},
$$

where

$$
\begin{gathered}
A_{0}=\left\{x \in H: V(x) \geq V_{1}^{*}\right\} ; \\
A_{j}=\left\{x \in H: \frac{7}{8} V_{j}^{*}<V(x) \leq V_{j}^{*}\right\}, \quad j=1,2, \ldots, i .
\end{gathered}
$$

For $i \in \mathbf{Z}^{+}$we define $B_{i}$ by $B_{i}=\left\{x \in H: V(x) \leq V_{i}^{*}\right\}$. We note that $B_{i} \backslash B_{i+1}$ differs from $A_{i}$ by a null set and so for almost every $x$ in $B_{i} \backslash B_{i+1}$ we have

$$
\frac{7}{8} V_{i}^{*}<V(x) \leq V_{i}^{*} \text {. }
$$

If $V(x)=0$ on a set of positive measure, then (1.3) implies that $U(\gamma)=0$ a.e. Hence we may assume that $V(x)=0$ on a set of measure zero. Then we have $\lim _{i \rightarrow \infty} \mu\left(B_{i}\right)=0$ and so $\lim _{i \rightarrow \infty} \int_{B_{i}}|f(x)| d \mu=0$. Let $\alpha=\int_{B_{1}}|f(x)| d \mu \geq 1 / 2$. By applying Exercise 12.59 of E. Hewitt and K. Stromberg [4], we select a sequence $\left(D_{j}\right)_{1}^{\infty}$ of measurable subset of $B_{1}$ such that

$$
\left\{\begin{array}{lll}
\text { (i) } & D_{1}=B_{1} \\
\text { (ii) } & \int_{D_{j}}|f(x)| d \mu=2^{-j+1} \alpha & \\
\text { (iii) } & D_{j+1} \subset D_{j} & \text { for } j>1 \\
\text { (iv) } & \text { if } D_{j} \cap\left(B_{i} \backslash B_{i+1}\right) \neq \varnothing, & \text { then } j \in \mathbf{Z}^{+}
\end{array}\right.
$$


For $i \in \mathbf{Z}^{+}$, let $E_{i}=B_{i} \backslash B_{i+1}, F_{i}=D_{i} \backslash D_{i+1}$, and $W_{i}=\min \left(V_{i}^{*}, V_{i-1}^{*}-V_{i}^{*}\right)$, where $V_{0}^{*}=\infty$. Define the function $W$ on $G$ by

$$
W(x)= \begin{cases}V_{i, j}^{*} & \text { if } x \in E_{i} \cap F_{j}, i, j \in \mathbf{Z}^{+} \\ \infty & \text { otherwise }\end{cases}
$$

where $V_{i, j}^{*}=V_{i}^{*}+W_{i} / 2^{j+5}$. By (3.1) we have

$$
V<W \leq 2^{1 / 2} V \text { a.e. in } D_{1} .
$$

If $V_{i}^{*} \neq 0$, then we have

$$
\left\{\begin{array}{lll}
\text { (i) } & V_{i, j}^{*}<V_{i-1}^{*}<V_{i-1, k}^{*} & \text { for } k \leq j \text { and } i>1 \\
\text { (ii) } & V_{i, j}^{*}<V_{i, k}^{*} & \text { for } k<j \text { and } i \geq 1 .
\end{array}\right.
$$

Let $r_{0}=\infty$ and define $r_{j}=\sup \left\{W(x): x \in D_{j}\right\}$ for $j \in \mathbf{Z}^{+}$. It follows from (3.4) that

$$
\left\{\begin{array}{l}
\text { (i) } r_{1} \leq 2^{1 / 2} V_{1}^{*} \text { and }\left(r_{j}\right)_{1}^{\infty} \text { is strictly decreasing to zero; } \\
\text { (ii) } D_{j}=\left\{x \in G: W(x) \leq r_{j}\right\}, \quad j \in \mathbf{Z}^{+} ; \\
\text {(iii) } F_{j}=\left\{x \in G: r_{j+1}<W(x) \leq r_{j}\right\}, \quad j \in \mathbf{Z}^{+} .
\end{array}\right.
$$

Since $\|f\|_{1}=1$ and $\alpha \geq 1 / 2$, it follows from (3.2) and (3.5) that

$$
\left\{\begin{array}{l}
\text { (i) } \int_{G}|f(x)| d \mu \leq 4 \int_{F_{1}}|f(x)| d \mu ; \\
\text { (ii) } \int_{D_{j}}|f(x)| d \mu=4 \int_{F_{j+1}}|f(x)| d \mu \text { for } j \in \mathbf{Z}^{+} .
\end{array}\right.
$$

4. Proof of Theorem 1.2. Let $1<p \leq q \leq p^{\prime}$. We shall first prove that $\|\hat{f} U\|_{q} \leq C\|f V\|_{p}$ for all simple functions $f$ in $L_{1}(G)$. Without loss of generality we may assume that $\|f\|_{1}=1$. Following the proof of Theorem 1 in Muckenhoupt [7] we observe that $\|\hat{f} U\|_{q}^{q}$ is bounded by $2^{q}$ times the sum of

$$
\sum_{j=-\infty}^{\infty} \int_{2^{j / p^{\prime} B}<U^{\#}(\gamma) \leq 2^{(j+1) / p^{\prime} B}}\left|\left(f \xi_{V \geq 2^{j / p^{\prime}-1 / p}}\right)^{\wedge}(\gamma)\right|^{q} U(\gamma)^{q} d \lambda
$$

and

$$
\sum_{j=-\infty}^{\infty} \int_{2^{j / p^{\prime}} B<U^{*}(\gamma) \leq 2^{(j+1) / p^{\prime} B}}\left|\left(f \xi_{V<2^{j / p^{\prime}-1 / p}}\right)^{\wedge}(\gamma)\right|^{q} U(\gamma)^{q} d \lambda,
$$

where $U^{\#}$ is defined on $\Gamma$ by $U^{\#}(\gamma)=U(\gamma)\|\gamma\|^{1 / \beta}, \beta$ being defined by $1 / \beta=$ $1 / p+1 / q-1$. We shall prove that (4.1) and (4.2) are each bounded by $C\|f V\|_{p}^{q}$. To estimate (4.1) we note that it is bounded by

$$
\begin{aligned}
& \sum_{j=-\infty}^{\infty} \int_{2^{j / p^{\prime}} B<U^{\#}(\gamma) \leq 2^{(j+1) / p^{\prime} B}}\left|\left(f \xi_{V \geq 2^{j / p^{\prime}-1 / p}}\right)^{\wedge}(\gamma)\right|^{q}\left(2^{(j+1) 1 / p^{\prime}} B\|\gamma\|^{-1 / \beta}\right)^{q} d \lambda \\
& \leq B^{q} \sum_{j=-\infty}^{\infty} 2^{(j+1) q / p^{\prime}}\left(\sum_{n=-\infty}^{\infty} \int_{\Gamma_{n} \backslash \Gamma_{n-1}} \mid\left(f \xi_{\left.\left.V \geq 2^{j / p^{\prime}-1 / p}\right)\left.^{\wedge}(\gamma)\right|^{q}\left(m_{n}\right)^{\left(q / p^{\prime}-1\right)} d \lambda\right)}\right.\right. \\
& \leq C \sum_{j=-\infty}^{\infty} 2^{(j+1) q / p^{\prime}}\left[\int\left|\left(f \xi_{V \geq 2^{j / p^{\prime}-1 / p}}\right)(x)\right|^{p} d \mu\right]^{q / p}
\end{aligned}
$$


(by Onneweer [9, Theorem 3(b) with obvious modification] and the HausdorffYoung inequality for Lorentz spaces)

$$
=C\left[\int_{G} \mid f(x)^{p} h(x) d \mu\right]^{q / p}
$$

where

$$
h(x)=\sum_{j=-\infty}^{\infty} 2^{j p / p^{\prime}} \xi_{V \geq 2^{j / p^{\prime}-1 / p}}(x) \leq C V(x)^{p} .
$$

Hence (4.1) is bounded by $C\|f V\|_{p}^{q}$.

To estimate (4.2) we note that $f$ has a support of finite measure. Let $H=\{x \in$ $G: f(x) \neq 0\}$. Using the notation in $\S 3$, we have (4.2) bounded by

$$
\begin{aligned}
& \sum_{j=-\infty}^{\infty} \int_{2^{j / p^{\prime}} B<U^{\#}(\gamma) \leq 2^{(j+1) / p^{\prime} B}}\left[\int\left|\left(f \xi_{V<2^{j / p^{\prime}-1 / p}}\right)(x)\right| d \mu\right]^{q} U(\gamma)^{q} d \lambda \\
& \quad \leq \int_{\Gamma}\left[\int_{\left\{x \in H: V(x)<2^{-1 / p} U^{\#}(\gamma) / B\right\}}|f(x)| d \mu\right]^{q} U(\gamma)^{q} d \lambda \\
& \quad \leq \sum_{j=0}^{\infty}\left(\int_{B r_{j+1}<U^{\#}(\gamma) \leq B r_{j}} U(\gamma)^{q} d \lambda\right)\left(\int_{\left\{x \in H: V(x)<2^{-1 / p} r_{j}\right\}}|f(x)| d \mu\right)^{q} \\
& \quad=\mathrm{I}+\mathrm{II},
\end{aligned}
$$

where

$$
\mathrm{I}=\left(\int_{B r_{1}<U^{\#}(\gamma)} U(\gamma)^{q} d \lambda\right)\left(\int_{\{x \in H: V(x)<\infty\}}|f(x)| d \mu\right)^{q}
$$

and

$$
\mathrm{II}=\sum_{j=1}^{\infty}\left(\int_{B r_{j+1}<U^{\#}(\gamma) \leq B r_{j}} U(\gamma)^{q} d \lambda\right)\left(\int_{\left\{x \in H: V(x)<2^{-1 / p} r_{j}\right\}}|f(x)| d \mu\right)^{q} .
$$

By (3.6)(i) we have I bounded by

$$
\begin{aligned}
& \left(\int_{B r_{1}<U^{\#}(\gamma)} U(\gamma)^{q} d \lambda\right)\left(\int_{H}|f(x)| d \mu\right)^{q} \\
& \quad \leq 4^{q}\left(\int_{B r_{1}<U^{\#}(\gamma)} U(\gamma)^{q} d \lambda\right)\left(\int_{F_{1}}|f(x)| d \mu\right)^{q} .
\end{aligned}
$$

To estimate II, we note that $1<p \leq q \leq p^{\prime}$ implies that $1<p \leq 2$. It follows from (3.5)(i) that if $x \in H$ and $V(x)<2^{-1 / p} r_{j}$ for some $j>1$, then $x \in D_{1}=B_{1}=\left\{x \in H: V(x) \leq V_{1}^{*}\right\}$. Hence we have $\left\{x \in H: V(x)<2^{-1 / p} r_{j}\right\} \subset$ $\left\{x \in D_{1}: W(x)<r_{j}\right\} \subset D_{j}$ for $j \geq 1$. Thus II is bounded by

$$
\begin{aligned}
& \sum_{j=1}^{\infty}\left(\int_{B r_{j+1}<U^{\#}(\gamma)} U(\gamma)^{q} d \lambda\right)\left(\int_{D_{j}}|f(x)| d \mu\right)^{q} \\
& \left.\quad=4^{q} \sum_{j=1}^{\infty}\left(\int_{B r_{j+1}<U^{\#}(\gamma)} U(\gamma)^{q} d \lambda\right)\left(\int_{F_{j+1}}|f(x)| d \mu\right)^{q} \quad \text { (by }(3.6)(\mathrm{ii})\right)
\end{aligned}
$$


Hence we get

$$
\begin{aligned}
\mathrm{I}+\mathrm{II} \leq & 4^{q} \sum_{j=0}^{\infty}\left(\int_{B r_{j+1}<U^{\#}(\gamma)} U(\gamma)^{q} d \lambda\right)\left(\int_{F_{j+1}}|f(x)| d \mu\right)^{q} \\
= & 4^{q} \sum_{j=0}^{\infty}\left(\int_{B r_{j+1}<U^{\#}(\gamma)} U(\gamma)^{q} d \lambda\right)\left(\int_{F_{j+1}}|f(x)| V(x) V(x)^{-1} d \mu\right)^{q} \\
\leq & 4^{q} \sum_{j=0}^{\infty}\left(\int_{B r_{j+1}<U^{\#}(\gamma)} U(\gamma)^{q} d \lambda\right)\left(\int_{F_{j+1}} V(x)^{-p^{\prime}} d \mu\right)^{q / p^{\prime}} \\
& \times\left(\int_{F_{j+1}}|f(x)|^{p} V(x)^{p} d \mu\right)^{q / p} \quad(\text { by Hölder's inequality) } \\
\leq & C \sum_{j=0}^{\infty}\left(\int_{F_{j+1}}|f(x)|^{p} V(x)^{p} d \mu\right)^{q / p} \quad \text { (by (3.5)(iii), (3.3), and (1.3)) } \\
\leq & C\|f V\|_{p}^{q}(\text { since } q / p \geq 1) .
\end{aligned}
$$

Thus (4.2) is also bounded by $C\|f V\|_{p}^{q}$. Hence we have $\|\hat{f} U\|_{q} \leq C\|f V\|_{p}$.

Having proved that $\|\hat{f} U\|_{q} \leq C\|f V\|_{p}$ for all simple functions $f$ in $L_{1}(G)$, we now note that the class of all simple functions in $L_{1}(G)$ is dense in $L_{1}(G)$. Hence for each $f$ in $L_{1}(G)$ with $\|f V\|_{p}<\infty$, there exist a sequence $\left(f_{n}\right)_{1}^{\infty}$ of simple functions in $L_{1}(G)$ such that $\left\|f_{n}-f\right\|_{1} \rightarrow 0$ and $\left\|f_{n} V\right\|_{p} \rightarrow\|f V\|_{p}$ as $n \rightarrow \infty$. Thus we have $\|\hat{f} U\|_{q} \leq C\|f V\|_{p}$ for all $f$ in $L_{1}(G)$.

5. Proof of Theorem 1.3. Let $1<q^{\prime} \leq p \leq q$. With obvious modifications in the proof of Theorem 1.2, we see that (1.4) is a sufficient condition for

$$
\left\|\check{h} V^{-1}\right\|_{p^{\prime}} \leq C\left\|h U^{-1}\right\|_{q^{\prime}}
$$

to hold for all $h$ in $L_{1}(\Gamma)$. By duality of weighted $L_{p}$-spaces and Parseval's identity we have

$$
\|\hat{f} U\|_{q} \leq C\|f V\|_{p}
$$

for all $f$ in $L_{1}(G)$ with compact support. The proof is complete by noting that the class of all $f$ in $L_{1}(G)$ with compact support is dense in $L_{1}(G)$.

6. Proof of Theorem 1.4. Let $1<p \leq q<\infty$. We first prove the necessity. In view of Theorem 1.1 it is sufficient to prove (1.5)(ii). Let $N$ be any fixed integer. For $i \in \mathbf{Z}$ such that $i<N$, choose $a \in G_{i} \backslash G_{i+1}$ and let $f=\xi_{a+G_{N}}$. Then for $\gamma \in \Gamma_{N}$ we have $\hat{f}(\gamma)=\bar{\gamma}(a)\left(m_{n}\right)^{-1}$, where $\mu\left(G_{N}\right)=\left(m_{N}\right)^{-1}$ and $\bar{\gamma}$ is the conjugate of $\gamma$. Hence we have

$$
\begin{aligned}
U_{N}\left(m_{N}-m_{N-1}\right)^{1 / q}\left(m_{N}\right)^{-1} & =\left(\int_{\Gamma_{N} \backslash \Gamma_{N-1}}|\hat{f}(\gamma) U(\gamma)|^{q} d \lambda\right)^{1 / q} \\
& \leq\|\hat{f} U\|_{q} \leq C\|f V\|_{p}=C V_{i}\left(m_{N}\right)^{-1 / p}
\end{aligned}
$$

Thus $U_{N}\left(m_{N}-m_{N-1}\right)^{1 / q}\left(m_{N}\right)^{-1 / p^{\prime}} \leq C V_{i}$. Since $2 m_{N-1} \leq m_{N}$, we have $U_{N} m_{N}^{1 / q-1 / p^{\prime}} \leq C V_{i}$ for $i<N$. Since $N$ is arbitrary, (1.5)(ii) is proved. 
To prove the sufficiency, we first consider the case $1<p \leq q \leq p^{\prime}$. Recall that $V$ and $U$ are nonnegative radial functions on $G$ and $\Gamma$, respectively such that (1.5)(ii) is satisfied. Let

$$
B=\sup _{n \in \mathbf{Z}}\left(\sup _{i<n} V_{i}^{-1} U_{n}\left(m_{n}\right)^{1 / q-1 / p^{\prime}}\right) \text {. }
$$

Let $f$ be in $L_{1}(G)$ such that support of $\hat{f} \subseteq \Gamma_{N}$ for some $N \in \mathbf{Z}$. By Theorem 1.2, a sufficient condition for $\|\hat{f} U\|_{q} \leq C\|f V\|_{p}$ is that

$$
\sup _{r>0}\left(\int_{\left\{\gamma \in \Gamma_{N}: U^{\#}(\gamma)>B r\right\}} U(\gamma)^{q} d \lambda\right)^{1 / q}\left(\int_{V(x)<r} V(x)^{-p^{\prime}} d \mu\right)^{1 / p^{\prime}}<\infty,
$$

where $U^{\#}(\gamma)=U_{n}\left(m_{n}\right)^{1 / q-1 / p^{\prime}}$ if $\gamma \in \Gamma_{n} \backslash \Gamma_{n-1}, n \in \mathbf{Z}$. Let $r$ be any positive real number and let $k=\sup \left\{n \leq N: U_{n}\left(m_{n}\right)^{1 / q-1 / p^{\prime}}>B r\right\}$. If $k=-\infty$, then the left-hand side of (6.1) is zero. Hence we may assume that $k$ is finite. We note that if $V_{n}<r$, then $n \geq k$. Indeed, if there exists $j<k$ such that $V_{j}<r$, then (1.5)(ii) implies that $B r>B V_{j} \geq U_{k}\left(m_{k}\right)^{1 / q-1 / p^{\prime}}>B r$, a contradiction. Hence we have

$$
\begin{gathered}
\left(\int_{\left\{\gamma \in \Gamma_{N}: U^{\#}(\gamma)>B r\right\}} U(\gamma)^{q} d \lambda\right)^{1 / q}\left(\int_{V(x)<r} V(x)^{-p^{\prime}} d \mu\right)^{1 / p^{\prime}} \\
\leq\left(\int_{\Gamma_{k}} U(\gamma)^{q} d \lambda\right)^{1 / q}\left(\int_{G_{k}} V(x)^{-p^{\prime}} d \mu\right)^{1 / p^{\prime}} .
\end{gathered}
$$

By (1.5)(i), (6.1) is satisfied and so we have $\|\hat{f} U\|_{q} \leq C\|f V\|_{p}$.

Now let $f \in L_{1}(G) \cap L_{\infty}(G)$ such that $\|f V\|_{p}<\infty$. Applying the result obtained in the above paragraph we have $\left\|\left(\hat{f} \xi_{\Gamma_{n}}\right) U\right\|_{q} \leq C\left\|\left(f * e_{n}\right) V\right\|_{p}$ for $n \in \mathbf{Z}$, where $e_{n}=\mu\left(G_{n}\right)^{-1} \xi_{G_{n}}$ and $*$ denotes convolution. Taking the limit, we have $\|\hat{f} U\|_{q} \leq$ $C\|f V\|_{p}$. Since $L_{1}(G) \cap L_{\infty}(G)$ is dense in $L_{1}(G)$ we have $\|\hat{f} U\|_{q} \leq C\|f V\|_{p}$ for all $f$ in $L_{1}(G)$, where $1<p \leq q \leq p^{\prime}$.

For the case $1<q^{\prime} \leq p \leq q$, let $f$ be in $L_{1}(G)$ such that support of $f \subseteq G_{N}$ for some $N \in \mathrm{Z}$. Let $k=\inf \left\{i \geq N: V_{i}^{-1}\left(m_{i}\right)^{1 / q-1 / p^{\prime}}>B r\right\}$. We may assume that $k$ is finite. It follows from (1.5)(ii) that we have $n \leq k$ whenever $U_{n}^{-1}<r$. By Theorem 1.3 and an argument similar to the previous proof, we have $\|\hat{f} U\|_{q} \leq$ $C\|f V\|_{p}$. Since the class of all functions in $L_{1}(G)$ with compact support is dense in $L_{1}(G)$, we have $\|\hat{f} U\|_{q} \leq C\|f V\|_{p}$ for all $f$ in $L_{1}(G)$, where $1<q^{\prime} \leq p \leq q$.

ACKNOWLEDGMENT. The author would like to thank the referee for valuable suggestions regarding the presentation of this paper and the proof of Theorem 1.3.

\section{REFERENCES}

1. J. J. Benedetto and H. P. Heinig, Weighted Hardy spaces and the Laplace transform, Lecture Notes in Math., vol. 992, Springer-Verlag, Berlin and New York, 1982, pp. 240-277.

2. J. J. Benedetto, H. P. Heinig, and R. Johnson, Boundary values of functions in weighted Hardy spaces, preprint .

3. H. P. Heinig, Weighted norm inequalities for classes of operators, Indiana Univ. Math. J. 33 (1984), 573-582.

4. E. Hewitt and K. Stromberg, Real and abstract analysis, Springer-Verlag, Berlin and New York, 1985. 
5. W. B. Jurkat and G. Sampson, On rearrangement and weight inequalities for the Fourier transform, Indiana Univ. Math. J. 33 (1984), 257-270.

6. B. Muckenhoupt, Weighted norm inequalities for classical operators, Proc. Sympos. Pure Math., vol. 35, Amer. Math. Soc., Providence, R.I., 1979, pp. 69-83.

7. _ - Weighted norm inequalities for the Fourier transform, Trans. Amer. Math. Soc. 276 (1983), 729-742.

8. __ A note on two weight function conditions for a Fourier transform norm inequality, Proc. Amer. Math. Soc. 88 (1983), 97-100.

9. C. W. Onneweer, Generalized Lipschitz spaces and Herz spaces on certain totally disconnected groups, Lecture Notes in Math., vol. 939, Springer-Verlag, Berlin and New York, 1982, pp. 106-121.

10. $\ldots$, Weak $L_{p}$-spaces and weighted norm inequalities for the Fourier transform on locally compact Vilenkin groups, preprint.

Department of MAThematics, National University of Singapore, Kent RIDGE, SINGAPORE 0511, REPUBLIC OF SINGAPORE 